La última novela publicada en vida por el autor británico E. M. Forster, A Passage to India, que transcurre en la India

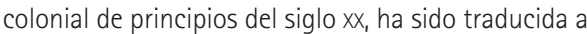
nuestro idioma en tres ocasiones. En el presente trabajo analizaremos la traducción del discurso de los personajes indios de la novela.

PALABRAS CLAVE: Traducción literaria, E. M. Forster, inglés, castellano, diálogos, India.

\title{
La traducción del discurso de los personajes indios en $A$ Passage to India de E. M. Forster*
}

\section{Marina Alonso Gómez Universidad de Málaga}

* Este trabajo se ha llevado a cabo gracias a la financiación del Ministerio de Educación, Cultura y Deporte..
The translation of the speech of the Indian characters in E. M. Forster's A Passage to India

The last novel published by British author E. M. Forster during his lifetime, A Passage to India, takes place in colonial India at the beginning of the 20th century. There are three different translations into Spanish of this novel, and in this paper we will analyse how these translations render the speech of the Indian characters.

KEY WORDS: Literary translation, E. M. Forster, English, Spanish, dialogues, India. 
GÉNESIS, RECEPCIÓN Y CRÍTICA DE $A$ PASSAGE TO INDIA

170 Forster viajó por primera vez a la India en I9I2. Partió de Nápoles junto con G. L. Dickinson y Robert C. Trevelyan a principios de octubre y, tras hacer escala en Port Said, llegaron a Bombay dos semanas después (Shahane (ed.), r989). Durante los siguientes meses, Forster recorrió el país y visitó a diversos amigos y conocidos, entre ellos Syed Ross Masood, al que el escritor había conocido seis años antes y con quien mantendría una amistad que duraría toda la vida (Furbank, I979); según el propio Forster, sería a raíz de esta amistad que escribiría la que suele considerarse su novela más importante, $A$ Passage to India: "Until I met [Masood], India was a vague jumble (...) and I was not interested in such a jumble (...) I dedicated [A Passage to India] to him out of gratitude as well as out of love, for it would never have been written without him», diría muchos años después (Stallybrass, I989:8). En el transcurso de su estancia en el subcontinente indio, Forster aprovechó todas las oportunidades que se le ofrecieron para conocer el país y sus habitantes, y algunas de sus experiencias, como su encuentro con un brahmán en Lahore, su visita a las Barabar Hills o la diatriba de uno de sus anfitriones indios contra el dominio inglés (Furbank, I979), se vieron después reflejadas de una u otra forma en las páginas de $A$ Passage to India. "It is odd that I should have seen so much of the side of life that is hidden from most English people», escribiría en The Hill of Devi (1953:37-38). Forster regresó a Inglaterra en abril de I9I3 con la idea de escribir una novela que se desarrollara en la India; sin embargo, después de escribir algunos capítulos no fue capaz de continuar y abandonó el proyecto (Furbank, I979). Mientras Forster se encontraba en Alejandría, donde trabajó para la Cruz Roja entre I9I5 y I9I8, el Maharajá de Dewas, al que había conocido en su primera estancia en la India, le escribió para ofrecerle un puesto en su corte (Furbank, I979). Forster hubo de rechazar la propuesta, pero, una vez de vuelta en Inglaterra, le pidió al Maharajá que renovara su oferta y en marzo de I92I viajó a la India por segunda vez (Furbank, I979). En esta ocasión permaneció en el país hasta enero de I922, y residió la mayor parte del tiempo en el estado nativo de Dewas, del que el Maharajá era soberano. Durante su estancia tuvo oportunidad de presenciar varios festivales hindúes, entre ellos el de Gokul Ashtami, que aparece descrito en detalle en la tercera sección de $A$ Passage to India; algunos otros incidentes descritos en la novela también fueron pergeñados a partir de experiencias vividas por el autor en esta segunda visita (Allen, I965). A finales de enero de 1922 regresó a Europa, previo paso por Egipto, y ya de vuelta en Inglaterra Leonard Woolf lo exhortó a terminar la novela que había comenzado al regresar de su primer viaje (Furbank, I979). Forster siguió los consejos de Woolf y escribió el grueso de lo que sería $A$ Passage to India a lo largo de r923 (Stallybrass, I989). La novela se publicó el 4 de junio de r924 y fue tanto aclamada por la crítica como un éxito entre el público (Stallybrass, r989; Childs, 2007). A finales de ese mismo año se habían editado I7 ooo ejemplares en Gran Bretaña y más de 54 ooo en Estados Unidos, la mayor parte de los cuales se vendieron (Stallybrass, I989), y había recibido alabanzas por parte de los críticos en ambos países y en la India, aunque no así en la India británica (Childs, 2007).

Los críticos suelen estar de acuerdo en que una de las características atribuibles a $A$ Passage to India es la amplia variedad de lecturas e interpretaciones de que ha sido objeto desde su publicación, cualidad que M. Sivaramakrishna 
(1989:5) describe como «its inexhaustibility to critical contemplation». Malcom Bradbury (1979) y José Ramón Losada Durán (I989) también coinciden en que la obra ha sido interpretada de formas muy diferentes, así como en señalar la dificultad que encuentran los críticos a la hora de determinar cuál de sus aspectos es el principal. En la misma línea, Meenakshi Mukherjee (I989) comenta que suele existir unanimidad en lo que a la pluralidad de niveles de significado de la novela se refiere, siendo la importancia relativa de los mismos lo que induce discrepancias entre la crítica. En opinión de Francisco Fernández (1988), la propia novela se presta a revelar nuevas claves con cada nueva lectura. Fernández (1988) también recuerda que la lectura política fue la primera que realizó la crítica, y tanto Lidan Lin (1997) como Tomasz Dobrogoszcz (2010) especifican que la interpretación más típica de la obra era la que la entendía como una crítica al imperialismo británico en la India. Esta interpretación no ha desaparecido por completo, pues aún en los años ochenta, noventa y principios del siglo Xxi encontramos autores que opinan que la novela retrata la presencia británica en la India de forma total y absolutamente negativa, como Jean Marc Moura (I996), o que algunos personajes indios constituyen figuras de resistencia al dominio colonial, como es el caso de Charu Malik (1997). Sin embargo, como bien indican Lin (1997) y Dobrogoszcz (20ro), la escuela poscolonialista vino a cuestionar esta lectura y proponer otras nuevas. En 1978, Edward W. Said mencionaba la novela de Forster en su obra Orientalism, en la que afirmaba que el desenlace de $A$ Passage to India refuerza la idea de que el Oriente estaba «destined to bear its foreignness as a mark of its permanente estrangement from the West», si bien «the gulf between [them] can be modulated (...) by superior Western knowledge and power» (Said, 1978:244). Desde entonces, muchos son los autores que han criticado la imagen de la India colonial que ofrece Forster en A Passage to India. Lidan Lin (1997), por ejemplo, encuentra que la caracterización de los personajes indios es negativa, mientras que la de los británicos, pese a sus defectos, apunta a sus cualidades como gobernantes, de manera que la superioridad inglesa vendría a justificar la presencia de Inglaterra en la India. Benita Parry (1978) considera que $A$ Passage to India se encuentra en los límites del discurso colonialista: si bien la novela critica el imperialismo, no por ello deja de ser heredera del discurso colonialista, además de obviar las dimensiones históricas del imperialismo. Peter Morey (2007) también detecta la presencia de la retórica imperialista en la novela, aunque cree que en ocasiones el uso que Forster hace de ella no es intencionado, y en su opinión el texto deja ver que el autor, a pesar de su simpatía hacia los nativos, no era capaz de identificarse plenamente con el anticolonialismo. En cambio Moura (1996:283), que no duda en calificar $A$ Passage to India de "classique de l'anti-colonialisme littéraire», considera que precisamente su originalidad radica en el uso que hace de las propias formas y premisas del discurso literario colonialista en contra del mismo.

En 1957 Forster escribió un prefacio para la edición de $A$ Passage to India publicada dentro de la serie Everyman's Library de la editorial Penguin Books. En dicho prefacio afirmaba que su intención principal al escribir la novela no era ni política ni sociológica, así como que quien quisiera descubrir cuál había sido podía encontrar una respuesta en la introducción que Peter Burra. Según Burra (1989), el verdadero tema de la novela era la amistad entre dos de los personajes principales, el director inglés Fielding y el médico indio Aziz, la cual, según algunos 
críticos, estaría basada en la relación entre Forster y Masood (Childs, 2007). Sin embargo, al igual que no pusieron fin a las lecturas en clave política, las declaraciones de Forster no impidieron que muchos críticos señalaran que uno de los temas fundamentales de la novela eran las relaciones sociales entre pueblos y culturas diferentes en general (Valdeón, I994-1995) y entre indios y británicos en particular (MartínezCabeza, I994), así como la separación existente entre ellos (Losada Durán, i989).

\section{LENGUA Y COMUNICACIÓN EN $A$ PASSAGE TO INDIA}

Bikram K. Das (I989) recoge esta idea como punto de partida para su trabajo «A Stylistic Analysis of the Speech of the Indian Characters in Forster's A Passage to India», publicado por primera vez en I975 como parte del volumen Focus on Forster's "A Passage to India": Indian essays in criticism, una obra cuyo objetivo consistía en venir a paliar la exigua participación india en la respuesta crítica y académica a la novela. Das señala que una de las posibles lecturas de A Passage to India es la de la interacción social entre la cultura india y la británica, tema cuyo elemento esencial sería el fracaso de la comunicación entre ambas y, a partir de esa premisa, aborda un estudio estilístico del discurso oral de los personajes indios de la novela. Cabe señalar que el de Das no es el único trabajo que se ha interesado por las interacciones orales y el uso del idioma entre los distintos personajes de A Passage to India: Miguel Ángel MartínezCabeza Lombardo (I994) aplica en uno de sus artículos el modelo de Brown y Levinson a los diálogos interculturales (esto es, entre uno o más personajes indios y uno o más personajes británicos) y Roberto Antonio Valdeón García (I994-I995), que coincide con Das en que el uso del idioma por parte de los personajes es merecedor de atención por el papel que desempeña en las relaciones entre británicos e indios (que a su parecer son el tema central de la obra), analiza las funciones sociales que cumple la lengua inglesa en la novela y llega a la conclusión de que la principal consiste precisamente en crear barreras entre los personajes.

Aunque Forster incluye un número considerable de palabras de origen nativo en la novela, todos los diálogos reproducidos en $A$ Passage to India se transcriben en inglés (cabría aquí señalar que en aquellas de sus novelas que transcurren parcialmente en Italia, esto es, en Where Angels Fear to Tread y A Room with a $V$ iew, sí que aparecen algunos enunciados en lengua italiana). No obstante, en ocasiones se menciona que algún personaje se expresa en un idioma diferente al inglés, aunque no se reproduzca el correspondiente enunciado; al parecer, Forster redujo tanto estas indicaciones como el número de términos nativos al revisar el manuscrito de la novela (Beer, I987). Por ejemplo, se dice que varios personajes ingleses se dirigen en urdu a interlocutores indios, generalmente a los criados: «Now and then she (...) practised her Urdu on the servant» (p. I2o); "You might tell your servant to bring my horse. He doesn't seem to appreciate my Urdu» (p. I05). También le habla en urdu Aziz a Fielding (al que siempre se ha dirigido en inglés hasta ese momento) cuando se reencuentran por primera vez después de que su amistad se viera truncada por un malentendido dos años antes; el médico indio emplea esta lengua para pedirle que no lo siga y anunciarle que no desea ser amigo de ningún inglés con objeto de que lo entiendan sus hijos, que se encuentran presentes durante la conversación. Asimismo se muestra cómo el sobresalto y la contrariedad provocados por un accidente de coche llevan a otro de los personajes indios a 
recurrir al árabe para expresar sus emociones. A pesar de su infrecuencia, estos ejemplos vienen a recordarnos que, pese a la ausencia de diálogos en lenguas diferentes al inglés, personajes británicos y personajes indios pertenecen a comunidades lingüísticas diferentes que se comunican en una lengua extranjera para una de ellas y que no todos los miembros de la comunidad dominan (en la novela se da a entender que este es el caso de los criados y los niños).

Más poder evocador tienen quizá los casos en los que se comenta específicamente el uso del idioma de los personajes, generalmente el que hacen los indios de la lengua inglesa. En el primero, un muchacho indio utiliza como excusa los malentendidos que provoca entre los alumnos el expresarse en la lengua inglesa que les obligan a aprender para justificar la información errónea que acaba de proporcionar sobre el estado de salud de uno de sus profesores. En el segundo caso, un grupo de indios se sonríe ante la forma de hablar inglés de un compatriota, el médico hindú Panna Lal, que el texto describe con expresiones como «of low extraction» $y$ «of ignoble origin».

«The schoolboy murmured that another boy had told him, also that the bad English grammar the Government obliged them to use often gave the wrong meaning for words, and so led scholars into mistakes» (p. 94)

«Agitated, but alert, he saw them smile at his indifferent English, and suddenly he started playing the buffoon» (p. 210)

En estos ejemplos se observa una imagen negativa del inglés hablado por los indios, lo que vendría a coincidir con la opinión vertida por Forster en su ensayo «India Again», incluido en el volumen Two Cheers for Democracy: «So it is not surprising that their English is poor. They have learnt it from Indians and practise it on
Indians» (citado en Das, 1989:83). Esto parece corroborar la opinión de Nirad C. Chaudhuri, quien en un artículo de 1954 afirmaba que, por lo general, los ingleses siempre se mostraban negativos respecto a cómo hablaban su idioma los indios, y que «in his kindly manner even $\mathrm{Mr}$. Forster ha[d] felt amused by [their] English» (Chaudhuri, I954:23). Es cierto que en «India Again» Forster añade: «English does get talked and gets interlarded in the oddest way with the Indian vernacular...» (Das, I989:83), sin embargo, en $A$ Passage to India, un personaje inglés como es Fielding defiende la vivacidad con la que se expresan en lengua inglesa los indios más jóvenes, aludiendo al mismo tiempo al hecho de que la mayor parte de sus compatriotas (en la novela se implica que prácticamente todos los ingleses son socios del mismo «club» de la ciudad, en el que no se admiten indios) responden a la descripción de Chaudhuri:

«"Everything ranged coldly on shelves was what I thought - I say, Mr. Fielding, is the stud going to go in?"

"I hae ma doots."

"What's that last sentence, please? Will you teach me some new words and so improve my English?"

Fielding doubted whether "everything ranged coldly on shelves" could be improved. He was often struck with the liveliness with which the younger generation handled a foreign tongue. They altered the idiom, but they could say whatever they wanted to say quickly; there were none of the babuisms ascribed to them up at the club» (pp. 54-55)

También podemos ofrecer un ejemplo en que Forster describe de manera crítica el uso que hace uno de los personajes británicos de una lengua extranjera, el urdu. Más concretamente, 
se trata de la esposa del más alto funcionario británico de la ciudad, y sus interlocutoras son un grupo de damas indias; la escena se produce durante una reunión social ofrecida por su marido a la que se han invitado excepcionalmente a algunos indios:

«She had learnt the lingo, but only to speak to her servants, so she knew none of the politer forms and of the verbs only the imperative mood». (p. 34)

No debería escapársenos que en casi todos estos ejemplos el comentario acerca del empleo del idioma establece una relación entre dicho uso y una u otra forma de lucha de poder. En uno se insinúa un interés por parte del Gobierno británico en que los nativos no aprendan el inglés correctamente, en otro se da a entender que los británicos tienen por costumbre representar el uso del inglés que hacen los indios bajo una luz negativa y el último pone en evidencia una relación de poder en la que una de las partes utiliza el idioma de la otra exclusivamente para darle órdenes. Por otra parte, Lin considera que los «bona fide English idioms» que usa Aziz cuando discute sobre política con Fielding al final de la novela es la forma que tiene Forster de desacreditar su postura nacionalista, pues ponen de manifiesto su «deferential habit of playing down Indianness in order to sound English» (Lin, I997:I44).

Como ya hemos mencionado, todos los diálogos reproducidos en $A$ Passage to India se encuentran transcritos en inglés, aunque, según autores como Valdeón (I994-I995), se sobrentiende que los intercambios entre ciertos personajes se producen en su lengua materna. Forster, por tanto, habría tenido por delante tanto la tarea de representar en inglés la forma en que los nativos hablan su lengua materna como la de representar la manera en que los nativos hablan en inglés. Según Das (I989), Forster no tenía interés en representar en inglés cómo los personajes indios se comunican entre ellos en sus propias lenguas, en parte porque para hacerlo le habría hecho falta conocer en cierta profundidad dichas lenguas y en parte porque se entiende que la comunicación dentro de un mismo grupo lingüístico es total: sería entre grupos lingüísticos diferentes en los que la comunicación podría fracasar. Por lo que se refiere a la representación del uso que los nativos hacen del inglés, Das opina que, a pesar de que tradicionalmente los autores británicos han tendido a ridiculizar el inglés hablado por los indios, la mayor parte de los personajes nativos de $A$ Passage to India hablan «fairly 'good' English» y describe los ocasionales intentos de Forster por representar cómo hablan en inglés los indios como «impressionistic» $\mathrm{y}$ «far les accurate than Kipling's» (I989:85). ${ }^{\text {I }}$

Entre las características atribuibles al inglés hablado en la India, Das (1989:84-85) incluye «confusion of the phonology of Standard English; its tendency to confuse registers (...); its use of archaic or obsolete forms (...); [and] unusual syntax and lexis (...)». Aunque su artículo se centra en las desviaciones que cometen los personajes indios por lo que respecta al estilo, ya que en su opinión su incidencia sobre la comunicación es mayor, Das (I989:86) también lista, sin entrar en detalle, lo que él considera «a few instances of the more obvious deviations in grammar, lexis and phonology» presentes en el discurso de los personajes indios de la novela. En primer lugar, Das

I En una reseña que apareció en el Springfield Sunday Republican poco después de la publicación de la novela se comenta que las intervenciones de los personajes indios se caracterizan por «a tang of the unfamiliar» $y$ «[the] occasional formal turn of speech no native-born Englishman would employ» (S. A., 1997:282). 
recoge una serie de intervenciones de Aziz, el principal personaje indio:

«Then we are in the same box," he said cryptically. "Then is the City Magistrate the entire of your family now?» (p. I6)

«Sir, excuse me, I did. I mounted my bike and it burst in front of the Cow Hospital». (p. 44)

«You'll jolly jolly well not forget those caves, will you?» (p. 67)

A continuación lista intervenciones de otros personajes nativos de mayor o menor relevancia para la trama:

«You will make yourself chip». (p. 28)

«The shorter lady, she is my wife» (...) «The taller lady, she is Mrs Das». (p. 34)

«Half one league onward». (p. 74)

«Even when the lady is so uglier than the gentleman?» (p. 194)

A los ejemplos enumerados por Das podríamos añadir algunas intervenciones del Dr. Panna Lal, el médico hindú cuyo inglés es tachado de «indifferent» (p. 21o) por el narrador y provoca sonrisas entre otros personajes indios:

«Dr. Aziz, Dr. Aziz, where you been?» (p. 48)

«How is stomach (...) how head?» (p. 93)

También podemos mencionar uno de los pocos casos en los que Forster representa ortográficamente la forma de expresarse de uno de sus personajes indios (al final de este trabajo comentaremos el caso de un personaje británico), en el que Aziz afirma que Mohammed Latif, pariente pobre que hace las veces de sirviente de la familia, no habla ni entiende el inglés (de nuevo se utiliza el manejo de este idioma como símbolo de superioridad, pues
Aziz pretende ridiculizar a Mohammed Latif frente a sus invitadas inglesas), a lo que este responde:

«You spick lie». (p. II5)

En el primer ejemplo, Aziz se está dirigiendo a otro de los personajes principales, Mrs. Moore, en respuesta a un comentario que le revela que es viuda, igual que él. De acuerdo con las notas de la edición de la novela a cargo de Stallybrass, se trata de una equivocación similar a la que comete Aziz unos capítulos después cuando dice que siempre está «in the bazaar» en lugar de «in the market-place» en el sentido de «in the public eye»; la expresión correcta en este caso sería «in the same boat». En el siguiente ejemplo, Aziz está discutiendo con un superior y utiliza la forma presente del verbo «bust» para referirse a un incidente acaecido la noche anterior, aunque, de acuerdo con el World Atlas of Varieties of English de la Universidad de Friburgo, lo que denominan «zero-past tense forms of regular verbs» es un rasgo extremadamente infrecuente en el inglés propio de la India. En el tercer ejemplo, Aziz le pide a otro de los personajes principales, Adela Quested, que no olvide su invitación a llevarla de excursión a las cuevas en las que se produce el episodio en torno al cual gira la novela. Según el diccionario Merriam-Webster, «jolly well» es una expresión que se emplea para enfatizar sentimientos como el enfado, la irritación o el descontento; además, de acuerdo con Gumperz et al. (1982:49), «[r]epetition or reduplication of words and larger utterance elements can be observed at several levels in Indian English discourse» $\mathrm{y}$ «[the] direct influence of Indian languages appears in the reduplication of single words to effect a slight change in meaning», un fenómeno que afectaría específicamente a los adverbios. 
El autor del cuarto ejemplo es un personaje secundario al que se describe en el texto como «[an] ill-bred upstart». Según Bansal y Harrison (I998), los hablantes de lenguas indias como el gujarati, el bengalí o el maratí no siempre mantienen la distinción entre /i:/ e /I/ sino que sustituyen la primera por la segunda, y la grafía «chip» parece indicar que el personaje acorta la duración vocálica de la palabra cheap al pronunciarla. El quinto ejemplo se corresponde con las palabras que emplea un caballero indio al presentar a su mujer y a su hermana durante la reunión ofrecida por el principal funcionario de la ciudad. El propio Das (I989) nos informa de que se trata de un ejemplo de un rasgo frecuente de la sintaxis del inglés hablado por los indios: la presencia de dos sujetos en aposición en una misma oración. En el siguiente ejemplo, el terrateniente musulmán más importante del distrito invita a un funcionario inglés y su futura prometida a dar un paseo en su coche, y pronuncia estas palabras antes de quedarse dormido. Según las ya mencionadas notas de la edición de Stallybrass, se corresponden con un verso de «The Charge of the Light Brigade» de Tennyson, "with the comic substitution of 'one' for 'a' and of 'onwards' for 'onward'» (I989: 345). En el séptimo ejemplo, en el que se observa la ausencia del determinante «much» y cuyo autor es uno de los asistentes al juicio contra Aziz por su supuesto intento de propasarse con Adela en las cuevas, surge en respuesta a las palabras del jefe de policía, que afirma durante su actuación como fiscal que «the darker races are physically attracted by the fairer, but not vice versa» ( $p$. I94).

En los dos siguientes ejemplos Panna Lal se dirige a Aziz, en uno para reprocharle el haber faltado a una cita y en otro para indagar acerca de su estado de salud cuando Aziz cae enfermo; distintos elementos se encuentran ausentes en sus intervenciones, como el verbo auxiliar en la primera y el determinante en la segunda. Según Davydova (20II), la omisión de have en construcciones de pretérito perfecto compuesto es un rasgo infrecuente pero presente en uno de sus corpus de estudio, el que identifica como «upper-mesolectal variety of Indian English»; por otra parte, de acuerdo con el World Atlas of Varieties of English de la Universidad de Friburgo, la duplicación de determinantes es un rasgo penetrante u obligatorio en esta variedad (también lo sería la ausencia de ciertos determinantes, en concreto los artículos). El último ejemplo se caracteriza por desviaciones tanto de tipo gramatical como semántico y fonético, este último encarnado ortográficamente en la grafía spick. La intervención pretende ser irónica, pues se produce en respuesta a un comentario de Aziz, que afirma que Mohammed Latif no sabe inglés y, cuando este contesta, añade: «I spick a lie! Oh, jolly good. Isn't he a funny old man?».

\section{TRADUCCIÓN DEL DISCURSO DE LOS PERSONAJES INDIOS}

Por lo que respecta a la traducción al español, A Passage to India ha sido vertida a la lengua española en tres ocasiones. En I955 la editorial argentina Sur publicó la primera traducción, titulada $E l$ paso a la India y realizada por el escritor y traductor italo-argentino Juan Rodolfo Wilcock. En r981, la editorial madrileña Alianza publicó la primera traducción española, realizada por el traductor madrileño José Luis López Muñoz y titulada en primera instancia Un viaje a la India y, más tarde, a partir de 1985 , reeditada bajo el título Pasaje a la India. Por último, en 2004 el diario $A B C$ editó una colección titulada Biblioteca del Viajero en la que se incluía una tercera traducción, también titulada Pasaje a la India y realizada por el escritor 
colombiano Juan Gabriel Vásquez. A continuación analizaremos cómo trasladaron al español los distintos traductores cada uno de los ejemplos anteriormente citados, pues consideramos que, como elemento de caracterización de los personajes, reviste importancia, especialmente en una situación de dominio colonial como la que describe el libro, en la que colonos y colonizados se comunican en una lengua materna para los primeros pero extranjera para los segundos.

Ejemplo I

A Passage to India, 1924

«Then we are in the same box,» he said cryptically «Then is the City Magistrate the entire of your family now?»

El paso a la India, 1955

—Entonces somos compañeros — dijo crípticamente-Y Y jel Magistrado Urbano es ahora toda su familia? (p. 23)

Un viaje a la India, I98I -Entonces estamos en el mismo caso — dijo Aziz, un tanto misteriosamente-. ¿Y ahora no tiene usted más familia que el Magistrado Municipal? (p. 30)

Pasaje a la India, 2004 —Entonces estamos en la misma caja — dijo Aziz crípticamente—. ¿Y el Magistrado Municipal es todo lo que queda de su familia? (p. 25)

Ejemplo 2

A Passage to India, 1924 Sir, excuse me, I did. I mounted my bike and it bust in front of the Cow Hospital.

Elpaso a la India, 1955

Discúlpeme, señor, pero así lo hice. Tomé la bicicleta y se me pinchó frente al Hospital de las Vacas. (p. 52)

Un viaje a la India, $198 \mathrm{I}$ Tendrá que excusarme, Mayor, pero sí que acudí. Iba en la bicicleta y se me pinchó una rueda delante del Hospital de las Vacas. (p. 7o)

Pasaje a la India, 2004

Excúseme, señor, pero sí lo hice. Monté en mi bicicleta, y se me pinchó una rueda delante del Hospital de las Vacas. (p. 55)

Ejemplo 3

A Passage to India, I924 You'll jolly jolly well not forget those caves, will you?

El paso a la India, $\mathrm{I} 955$ Supongo que no se olvidará por nada, por nada, de la invitación a las Cuevas, ¿no? (p. 74)

Un viaje a la India, $\mathrm{I} 98 \mathrm{I}$ ¿No se olvidará usted de las cuevas, verdad? (p. Ioo)

Pasaje a la India, $2004 \quad$ No se olvidará usted de las cuevas, ¿verdad? (p. 79)

Ejemplo 4

A Passage to India, 1924 You will make yourself chip.

El paso a la India, 1955 Así se rebaja ante ellos. (p.74)

Un viaje a la India, I98I Se rebajará usted. (p. 48)

Pasaje a la India, $2004 \quad$ Se rebajará usted. (p. 39) 
Ejemplo 5

A Passage to India, $\mathrm{I} 924$

178
The shorter lady, she is my wife, she is Mrs Bhattacharya (...) The taller lady, she is my sister, she is Mrs Das.

El paso a la India, 1955 La dama más baja es mi esposa, es la señora Batacharia (...) La dama más alta es mi hermana, es la señora Das. (p. 42)

Un viaje a la India, $198 \mathrm{I}$ La señora más baja es mi mujer, Mrs. Bhattacharya (...) La señora más alta es mi hermana, Mrs. Das. (p. 56)

La dama más baja es mi mujer, la señora Bhattacharya (...)

Pasaje a la India, 2004

Ejemplo 6

\begin{tabular}{ll}
\hline A Passage to India, I924 & Half one league onwards! \\
\hline El paso a la India, I955 & Una media legua. (p. 82) \\
\hline Un viaje a la India, I98I & ¡Media legua de camino! (p. III) \\
\hline Pasaje a la India, 2004 & ¡Media legua hacia delante! (p. 87)
\end{tabular}

Ejemplo 7

A Passage to India, I924 Even when the lady is so uglier than the gentleman?

El paso a la India, I955 ¿Aun cuando la dama es tanto más fea que el caballero? (p. 204)

Un viaje a la India, I98I ¿Aunque la señora sea mucho más fea que el caballero? (p. 275)

Pasaje a la India, 2004 ¿Aunque la señora sea mucho más fea que el caballero? (p. 213)

Ejemplo 8

A Passage to India, I924 How is stomach, how head?

El paso a la India, I955 ¿Cómo anda estómago, cómo anda cabeza? (p. Ioo)

Un viaje a la India, I98I ¿Qué tal el estómago, qué tal la cabeza? (p. 135)

Pasaje a la India, 2004 ¿Qué tal el estómago, qué tal la cabeza? (p. Io7)

Ejemplo 9

\begin{tabular}{ll}
\hline A Passage to India, I924 & Dr. Aziz, Dr. Aziz, where you been? \\
\hline El paso a la India, I955 & Doctor Aziz, doctor Aziz, ¿dónde estuvo usted? (p. 56) \\
\hline Un viaje a la India, I98I & Doctor Aziz, doctor Aziz, ¿dónde estaba usted? (p. 76) \\
\hline Pasaje a la India, 2004 & Doctor Aziz, doctor Aziz, ¿dónde ha estado usted? (p. 6o) \\
\hline
\end{tabular}


Ejemplo ıо

\begin{tabular}{ll}
\hline A Passage to India, I924 & You spick lie. \\
\hline El paso a la India, I955 & Usted dici mentira. (p. I23) \\
\hline Un viaje a la India, I98I & Hablas una mentira. (p. I63) \\
\hline Pasaje a la India, 2004 & Hablas una mentira. (p. I27) \\
\hline
\end{tabular}

En la mayoría de los casos los tres traductores optan por traducir estas intervenciones sin reflejar sus particularidades, aunque existen excepciones en las que las traducciones se esfuerzan por reproducirlas. En el primer caso, por ejemplo, la traducción de 2004 opta por traducir literalmente la expresión empleada por Aziz («to be in the same box») para así transmitir el carácter críptico que el propio texto le otorga explícitamente. Tanto la traducción de r955 como la de I98I se muestran más convencionales en sus opciones, si bien la primera resulta menos transparente que la segunda, que de hecho se ve obligada a modular el «criptically» del original con un «un tanto misteriosamente» precisamente porque la frase resultante en castellano es notablemente menos oscura que la original. En el tercer y en el quinto ejemplos, la traducción de I955 es la única que trata de reproducir el efecto del original en castellano; para ello recurre en un caso a la repetición de la locución adverbial «para nada» y en el otro, a la del verbo «ser», anteponiendo este a lo que de otra forma habría sido una aposición más natural en castellano. En el séptimo ejemplo, resulta improbable que el uso no estándar del cuantificador «tanto» como expresión diferencial en la construcción comparativa de desigualdad que se observa en la traducción de I955 sea intencionado, pues también aparece en el discurso de personajes ingleses y del propio narrador.
En el siguiente ejemplo, la traducción de I955 replica la ausencia de determinantes posesivos del original mediante la ausencia de artículos definidos. Las traducciones de I98I y 2004 prescinden del verbo, lo que genera cierto coloquialismo. En el último ejemplo, la traducción de 1955 opta por un verbo idiomático pero mal conjugado o pronunciado, «dici», ${ }^{2}$ y por calcar la presencia de un objeto directo singular carente de artículo, «mentira»; las traducciones de r98I y 2004 optan en cambio por una estructura sintácticamente correcta con un verbo que, al igual que ocurría en el texto original, no es el que más frecuentemente aparece en colocación con el sustantivo «mentira», esto es, «hablar» en lugar de «decir» o «contar». En la mayor parte del resto de ejemplos no puede detectarse un esfuerzo consciente en las traducciones al español por reproducirse las peculiaridades o matices del texto original. En todos estos casos las traducciones al castellano son gramaticalmente correctas, así como idiomáticas. En algunos puede argumentarse que algunas resultan más naturales que otras, sin embargo, no parece probable que por sí solas sean capaces de despertar

2 Es posible que el traductor (cuya familia materna era de origen italo-parlante y que terminó por establecerse en Italia, adquirir la nacionalidad italiana y adoptar el italiano como lengua literaria y de traducción) esté aquí tratando de imitar con el uso de una forma verbal italiana las interferencias de la lengua materna que, según Das, se producen en los hablantes indios de inglés. 
en el lector la consciencia de estar leyendo la representación de una forma particular de habla.

Comentaremos por último un caso en el que el enunciante no es indio sino británico, pero cuya traducción al español reviste especial interés y en el que entra de nuevo en juego la percepción del dominio con el que los indios manejan la lengua inglesa.

\begin{tabular}{ll}
\hline A Passage to India, I924 & "I hae ma doots". (p. 55) \\
\hline El paso a la India, I955 & $\begin{array}{l}\text {-Lo dudo, lo dudo. (p. } \\
\text { 62) }\end{array}$ \\
\hline & $\begin{array}{l}\text {-Tengo mis dudas - } \\
\text { respondió el otro, imi- } \\
\text { tando el acento que suele } \\
\text { an viaje a la India, } \\
\text { I98I }\end{array}$ \\
& $\begin{array}{l}\text { en las obras de teatro. } \\
\text { (p. 84) }\end{array}$ \\
\hline & $\begin{array}{l}\text {-Tengo mis dudas - } \\
\text { dijo Fielding con acento } \\
\text { Pasaje a la India, 2004 actor escocés. (p. 67) }\end{array}$ \\
\hline
\end{tabular}

En las notas de la edición de Stallybrass de I989 se aclara que "I hae ma doots' is how a stage Scotsman says 'I have my doubts'» (I989: 343). Fielding (que en el resto de la novela se expresa en inglés), dirige esta frase a Aziz, que, al no comprenderla, le pide que se la explique y, a renglón seguido, que le enseñe palabras nuevas para mejorar así su dominio de la lengua (esto, a su vez, da pie a las reflexiones de Fielding sobre el uso que hacen los indios del inglés que ya tuvimos ocasión de comentar). La traducción de 1955, en el mejor de los casos, da lugar a confusión, puesto que Aziz parecería no ser capaz de comprender una frase en inglés que en castellano no presenta ninguna dificultad y, en el peor, resulta incoherente, pues la intervención de Fielding no parece dar pie alguno a la respuesta (en forma de pregunta) de Aziz: «¿Qué quiere decir eso, por favor? ¿Me enseñará algunas palabras nuevas para mejorar mi inglés?». Las traducciones de 1981 y de 2004 suplen de manera explícita una información que se encuentra implícita en el texto original (o proporcionada en forma de notas en ediciones posteriores del mismo), esto es, que Fielding está imitando el habla escocesa. Además, según la traducción de 2004 se trata del acento propio de los actores escoceses, mientras que según la de I98I no tiene por qué tratarse de actores escoceses, sino de cualquier actor representando un personaje escocés, y el acento no tiene por qué ser realista.

\section{CONCLUSIONES}

La forma de expresarse de los personajes de una obra de ficción puede cumplir diferentes cometidos, la mayor parte de ellos relacionados con la caracterización del personaje en cuestión, entre ellos, mostrar el nivel social o educativo del hablante, indicar su procedencia geográfica o incluso reforzar características de su personalidad mediante el uso de determinados rasgos (palabras malsonantes, expresiones arcaicas, extranjerismos innecesarios, etc.). En el caso de una obra como $A$ Passage to India, cuya acción se desarrolla en un escenario colonial en el que colonos y colonizadores se comunican en un idioma que es lengua materna del primer grupo pero extranjera para los segundos, con el consiguiente, a priori, desequilibrio de competencia lingüística, que viene a reflejar el desequilibrio de poder inherente a toda situación de colonización, la importancia atribuible a la forma de expresarse de los personajes es todavía mayor. Como ya hemos visto, la propia novela ofrece en más de una ocasión comentarios acerca del uso del lenguaje de sus personajes y establece vínculos entre dicho uso y la posición que estos ocupan dentro del entramado social de la India colonial. 
En las traducciones de la obra disponibles en nuestro idioma, es frecuente que no se trasladen las particularidades y características del habla de los personajes indios en la novela, bien porque los traductores las pasaran por alto, bien porque no consiguieran dar con una fórmula con la que reproducirlas en castellano, bien porque no consideraran necesario mantenerlas en la lengua de destino. No obstante, también encontramos casos en los que las traducciones se esfuerzan por transmitirlas, para lo cual se adoptan distintas estrategias, como traducir literalmente las expresiones del original («estar en la misma caja» por «be in the same box»), calcar sus peculiaridades («Cómo anda estómago?» por «How is head?») o transponerlas a otros elementos de la oración («La dama más baja es mi esposa, es la señora Batacharia»). Sin embargo, salvo casos muy puntuales como es el del penúltimo ejemplo, en el que los comentarios de Aziz acerca de las palabras de Mohammed Latif ponen de relieve lo particular de las mismas (lo que también ocurre con la intervención a la escocesa de Fielding), es posible que el lector hispanohablante no capte el hecho de que el autor está tratando de reflejar lo que él considera el uso que los indios hacen del inglés.

Esto supondría una pérdida en lo que respecta a la caracterización de los personajes y, sobre todo, a la ambientación de la acción en un contexto histórico concreto como es el de la India colonial. En las ocasiones donde el propio texto comenta el uso que los personajes hacen del lenguaje, pueden incluso producirse incongruencias. Así, varios personajes se muestran divertidos ante el dominio de la lengua inglesa del que hace gala el Dr. Panna Lal, dominio que el texto original califica de «indifferent» (la traducción de 1955 traduce este adjetivo como «dudoso» y la de 2004 opta por «mediocridad», mientras que la de i98 I lo omite, aunque la implicación se mantiene), cuando en las traducciones al español la totalidad o la mayoría de las intervenciones de Panna Lal han sido correctas hasta ese momento. Algo similar, si bien en este caso no afecta al discurso en lengua inglesa de un personaje indio (aunque, en la traducción al castellano, sí a su nivel de comprensión de la misma), es lo que ocurre cuando en la primera de las traducciones a nuestro idioma se elimina cualquier indicio de que uno de los personajes está expresándose en escocés, dando lugar a la incongruencia de que otro personaje no lo entienda a pesar de no dar muestras en ningún otro momento de tener problemas en comprender la lengua inglesa. La situación resulta especialmente incongruente al tratarse de una expresión muy sencilla («I have my doubts» en inglés, «Lo dudo, lo dudo» en la traducción castellana) que difícilmente podría dar lugar a confusión.

Una posibilidad habría sido que las traducciones hubieran trasladado las particularidades del discurso de los personajes (en el caso que nos ocupa, más concretamente de los personajes indios), que se esfuerzan por transmitir en algunas pero contadas ocasiones, de manera más consistente, esto es, siempre que aparecen en el texto original. Para ello, podrían haber recurrido a las estrategias que ya emplean en algunos casos, como la traducción literal, el calco o la introducción de amplificaciones explicativas de información que se encuentra implícita en el texto original (estrategia que se emplea en la segunda y tercera traducciones cuando Fielding utiliza una expresión escocesa). De esta forma, los lectores de habla española habrían sido más conscientes del hecho de que, a pesar de que los personajes indios de la novela se expresan en inglés, esta no es su lengua materna sino la de sus colonizadores, con todas las implicaciones que esta situación conlleva. Esto sería parti- 
cularmente deseable en los casos en los que el propio texto comenta el uso del lenguaje de los personajes, para evitar así incoherencias entre la dicción de los personajes y los comentarios del narrador sobre la misma.

RECIBIDO EN SEPTIEMBRE DE 2017 ACEPTADO EN OCTUBRE DE 2018 VERSIÓN FINAL DE OCTUBRE DE 2018

\section{REFERENCIAS BIBLIOGRÁFICAS}

Allen, Glen O. (I955): «Structure, Symbol, and Theme in E. M. Forster's A Passage to India», PMLA, 70/5, 934-954.

Asociación de Academias de la Lengua EspaÑOLA (2OIO): NUEVA GRAMÁTICA DE LA LENGUA ESPAÑOLA, Madrid: Espasa.

Bansal, R. K y Harrison, J. B. (I998) [1972]: Spoken English, Hyderabad: Orient Longman.

BeEr, John (1987): "Conclusion: A Passage to India and the Versatility of the Novel», en John BEER (ed.), A Passage to India: Essays in interpretation, Basingstoke: Macmillan, I32-I52.

Bradbury, Malcom (1979): «Introduction», en: Malcom Bradbury (ed.), A Passage to India: A selection of critical essays. Londres: McMillan, II-27.

Burra, Peter (1983) [1957]: «Introduction to the Everyman edition", en E. M. Forster, $A$ Passage to India, Harmondsworth: Penguin, 319-333.

Chaudhuri, Nirad C. (1954): «Passage to and from India", Encounter, 2, I9-24.

Childs, Peter (2007): "A Passage to India», en David Bradshaw (ed.), The Cambridge Companion to E. M. Forster, Cambridge: Cambridge University Press, I88-208.

Davydova, Julia (20II): The Present Perfect in nonNative Englishes: A Corpus-Based Study of Variation, Berlín, Nueva York: De Gruyter Mounton.

DAs, Bikram K. (1989) [1975]: «A Stylistic Analysis of the Speech of the Indian Characters in Forster's A Passage to India», en Vasant A. Shahane (ed.), Focus on Forster's "A Passage to India". Indian essays in criticism, Hyderabad: Orient Longman, 78-88.

Dobrogoszcz, Tomasz (20I0): "A Passage to OUBOUM: Homi Bhabha reads E. M. Forster», en
Krzysztof Fordonski (ed.), Vasorvia: Instytut Kulturologii i Lingwistyki Antropocentrycznej, Uniwersytetu Warszawskiego and International E. M. Forster Society, 69-89.

Fernández, Francisco (I988): «A Passage to India: el lenguaje artístico y simbólico de E. M. Forster», Revista alicantina de estudios ingleses, I, 33-79.

Forster, Edward Morgan (1953): The Hill of Devi, Nueva York: Harcourt, Brace and Co.

- (I955): El paso a la India, trad. Juan Rodolfo Wilcock, Buenos Aires: Sur.

- (I98I): Un viaje a la India, trad. José Luis López Muñoz, Madrid: Alianza.

- (I989) [I957]: «Prefatory Note to the Everyman edition», en E. M. Forster, A Passage to India, Harmondsworth: Penguin, 317-318.

- (2004): Pasaje a la India, trad. Juan Gabriel Vásquez, Barcelona: Folio.

- (2015) [1924]: A Passage to India, Londres: Penguin.

Furbank, Philip N. (i979): E. M. Forster: A life, Oxford: Oxford University Press.

Gumperz, John J.; Aulakh, Gurinder y Kaltman, Hannah (I982): "Thematic structure and progression in discourse», en John J. Gumperz (ed.), Language and Social Identity, Cambridge: Cambridge University Press, 22-56.

«JOLLY WELl» (2018): MERRLAM-WEBSTER, <https:// www.merriam-webster.com/dictionary/jolly\%2o well> [consulta: 26-IX-20I8].

Kortmann, Bernd y Kerstin Lunkenheimer (eds.) (2013): The Electronic World Atlas of Varieties of English. Leipzig: Max Planck Institute for Evolutionary Anthropology, <http://ewave-atlas.org> [consulta: 26-IX-2018].

Lin, Lidan (I997): "The irony of colonial humanism: A Passage to India and the politics of the posthumanism", ARIEL: A Review of International English Literature, 38 /4, I33-I53.

Losada Durán, José Ramón (I989): «Nationalism and Imperialism in E. M. Forster's $A$ Passage to India», Anglo-American Studies, 9/2, I53-I5.

Malik, Charu (i997): "To express the subject of friendship: Masculine desire and colonialism in A Passage to India», en Robert K. Martin y George Piggford (eds.), Queer Forster, Chicago: University of Chicago Press, 22I-236.

Martínez-Cabeza Lombardo, Miguel Ángel (I994): «Conversations between British and 
Indian characters in $A$ Passage to India: a reappraisal of politeness theory», Revista Canaria de Estudios Ingleses, 29, 197-206.

Morey, Peter (2007): «Postcolonial Forster», en David Bradshaw (ed.), The Cambridge Companion to E. M. Forster, Cambridge: Cambridge University Press, 254-273.

MourA, Jean Marc (I996): «Le renversement du réalisme colonial dans $A$ Passage to India d'E. M. Forster», Études anglaises, 49/3, 283-294.

Mukherjee, Meenakshi (I989) [I975]:: «On Teaching $A$ Passage to India», en Vasant A. Shahane (ed.), Focus on Forster's "A Passage to India". Indian essays in criticism, Hyderabad: Orient Longman, 98-IO4.

PARRY, Benita (I978): «The politics of representation in $A$ Passage to India», en John Beer (ed.), A Passage to India: Essays in interpretation, Basingstoke: Macmillan, 27-43.

S. A. (1997): "Review, Springfield Sunday Republican», en Philip GARDNER (ed.), The Critical Heritage Series: E.M. Forster, Londres: Routledge, $28 \mathrm{I}-283$.
SAID, Edward W. (1978): Orientalism, Londres: Routledge and Paul Kegan.

Shahane, Vasant A. (ed.) (I989) [I975]: «Itinerary of Forster's visits to India», en Vasant A. Shahane (ed.), Focus on Forster's "A Passage to India". Indian essays in criticism, Hyderabad: Orient Longman, VII-XII.

SivARAMAKRISHNA, M. (I989) [I975]: «Marabar Caves Revisited», en Vasant A. Shahane (ed.), Focus on Forster's "A Passage to India". Indian essays in criticism, Hyderabad: Orient Longman, 5-I7.

Stallybrass, Oliver (1989): «Editor's introduction», en E. M. Forster, A Passage to India, Harmondsworth: Penguin, 7-28.

V. V. A. A. (1989). «Notes», en E. M. Forster, $A$ Passage to India, Harmondsworth: Penguin, 337-359.

VALDEón, Roberto Antonio (I994-I995): «Funciones sociales del inglés en $A$ Passage to India», Archivum: Revista de la Facultad de Filología, 44-45/2, I49-I72. 